\title{
Outre : Note linguistique complémentaire (Noms berbères)
}

Salem Chaker

\section{(2) OpenEdition}

1 Journals

Édition électronique

URL : https://journals.openedition.org/encyclopedieberbere/2877

DOI : $10.4000 /$ encyclopedieberbere. 2877

ISSN : 2262-7197

Éditeur

Peeters Publishers

\section{Édition imprimée}

Date de publication : 2 juin 2013

Pagination : 5999-6004

ISBN : 978-2-7584-0194-0

ISSN : 1015-7344

Référence électronique

Salem Chaker, «Outre : Note linguistique complémentaire (Noms berbères) », Encyclopédie berbère [En ligne], 36 | 2013, document 058, mis en ligne le 12 mars 2021, consulté le 17 février 2022. URL : http:// journals.openedition.org/encyclopedieberbere/2877 ; DOI : https://doi.org/10.4000/ encyclopedieberbere.2877

Ce document a été généré automatiquement le 17 février 2022.

(c) Tous droits réservés 


\title{
Outre : Note linguistique complémentaire (Noms berbères)
}

\author{
Salem Chaker
}

1 Les dénominations berbères de l'outre sont diversifiées mais, pour plusieurs d'entre elles, communes à l'ensemble du domaine. Elles distinguent généralement, et pas uniquement chez les nomades Touaregs, l'outre à eau, l'outre à denrées sèches, l'outrebaratte à lait, éventuellement l'outre à huile, à beurre frais, la vieille outre... Différenciations qui peuvent être considérées comme un indice net de l'importance de ce type de contenants et de celle de l'élevage (pastoral ?) et des technologies qui en découlent dans les sociétés berbères anciennes.

\section{En touareg}

2 Comme attendu chez un groupement de nomades éleveurs, le touareg dispose d'un vocabulaire de l'outre riche et bien différencié (on indique d'abord les forme de la tamâhaq, puis celles du touareg méridional : TM) :

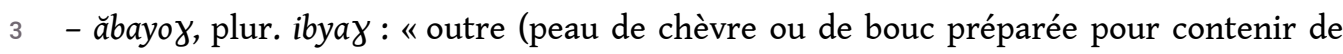
l'eau) ». tăbayoq (</tăbayoyt/) : « petite outre » (Foucauld, I, p. 44)

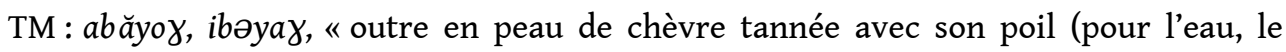
beurre, la farine) (Prasse et al., I, p. 60).

4 Racine BYy ; à noter que ce mot, avec cette signification, est spécifique au touareg où il désigne aussi une "ampoule, vessie, poche placentaire... ». Cela semble indiquer une signification première plus générale que « outre ». Le Maroc (chleuh, tamazight, rifain) connait une forme abyac, qui a des significations diverses : « sac en cuir, musette, sac de femme, outre, ventre femme enceinte... » (Naï-Zerrad 1998, p. 147), qui confirment un sens de base non spécialisé. Une correspondance touareg $/ \gamma />$ Maroc $/ \varepsilon /$ n'est pas courante mais reste possible (cf. chleuh ArEAT, «chameau", provenant de alyem), surtout pour un terme qui parait bien avoir de fortes connotations expressives. 
5 - ăğiwìr, iğiwār : « vieille outre » (Foucauld, I, p. 468)

TM : ne semble pas attesté. Racine GWR.

6 - ağədud, iğdad: "vieille outre en peau mince et sans poils (vieille tanwārt presque usée » (Foucauld, I, p. 395). Souvent utilisée pour contenir les dattes pilées.

TM : agădod, igədad / tagădott, tigədad, « vieille outre usée » (Prasse et al. I, p. 199). Racine GDD.

7 - anwar, inwāron : " outre en peau mince sans poils... sert à mettre le lait » (Foucauld, III, p. 1395)

TM : anwar / tanwart, « petite outre, en peau tannée et sans poils ; outre à lait » (Prasse II, p. 633). Racine NWR.

8 - talukkit, tilukkātīn : « outre à beurre » (Foucauld, III, p. 1031) ; donné, sans doute à tort, par Foucauld comme emprunté à l'arabe.

TM : ne semble pas attesté, mais le verbe alek, " ê. suspendu ", auquel talukkit pourrait être rattaché, est commun à l'ensemble de l'aire touarègue (Prasse et al, I, p. 470). Racine LK.

\section{En berbère Nord}

On y distingue fondamentalement entre :

10 - «Outre pour denrées sèches : grains, figues, semoule, farine » (en peau de mouton débarrassée de sa laine) :

tayləwt/taylut, masc. ayləw : Kabyle (Dallet, p. 920), Ouargla (Delheure, p. 243). Racine YLW.

awlək / iwlkan, tawləkt / tiwlkin Chleuh (Destaing, p. 207) ; tawlikt, tirukt, Rifain (Renisio, p. 439). Racine WLK.

11 et

12 - «Outre pour liquides, eau ou huile » (en peau de bouc, garnie de ses poils) :

Ayddid / iyddidən, est attesté en Kabyle (Dallet, p. 919) ; Chleuh (Destaing, p. 207) ;

Tamazight (Mercier, p. 182) ; Rifain (Renisio, p. 439).

Certains parlers présentent une forme aǧəddid, agəddid: Mzab (Delheure, p. 67), Ouargla (Delheure, p. 94), à relier au touareg agedud. Racine YDD / GDD.

13 Plusieurs dialectes distinguent :

14 - « Outre-baratte à lait :

tagnart / tignarin, Tamazight (Mercier, p. 182) ; Racine GNR (< WNR/NWR).

tawwart/ tiwwarin, Ouargla (Delheure, p. 228); formes qui renvoient au touareg

tanwart (même sens). Racine NWR > WWR.

tagəššult/tig ${ }^{w} \partial s ̌ l i n$, Chleuh (Destaing, p. 207).

15 - « Outre à huile »

abəțtan, ibəțțann, Tamazight (Mercier, p. 182).

agərḍ, igərḍan, tagərțt, tigerḍin, Chleuh (Destaing, p. 207).

16 - « Outre à beurre frais » : aslix, islixən, Ouargla (Delheure, p. 188).

17 - « Outre vieille et usée » : agərbuz / agərbaz, « vieille outre usée » Tamazight (Mercier,

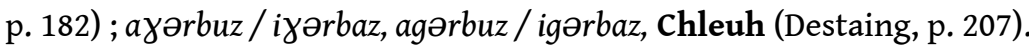


Il existe de nombreuses autres dénominations locales d'outres particulières, par la taille ou la destination: par ex.: Ouargla : ašibuțt, tišibuțin, "petite outre » (Delheure, p. 206)....

On retiendra que l'on peut identifier au moins trois racines largement répandues dans l'ensemble du domaine berbère :

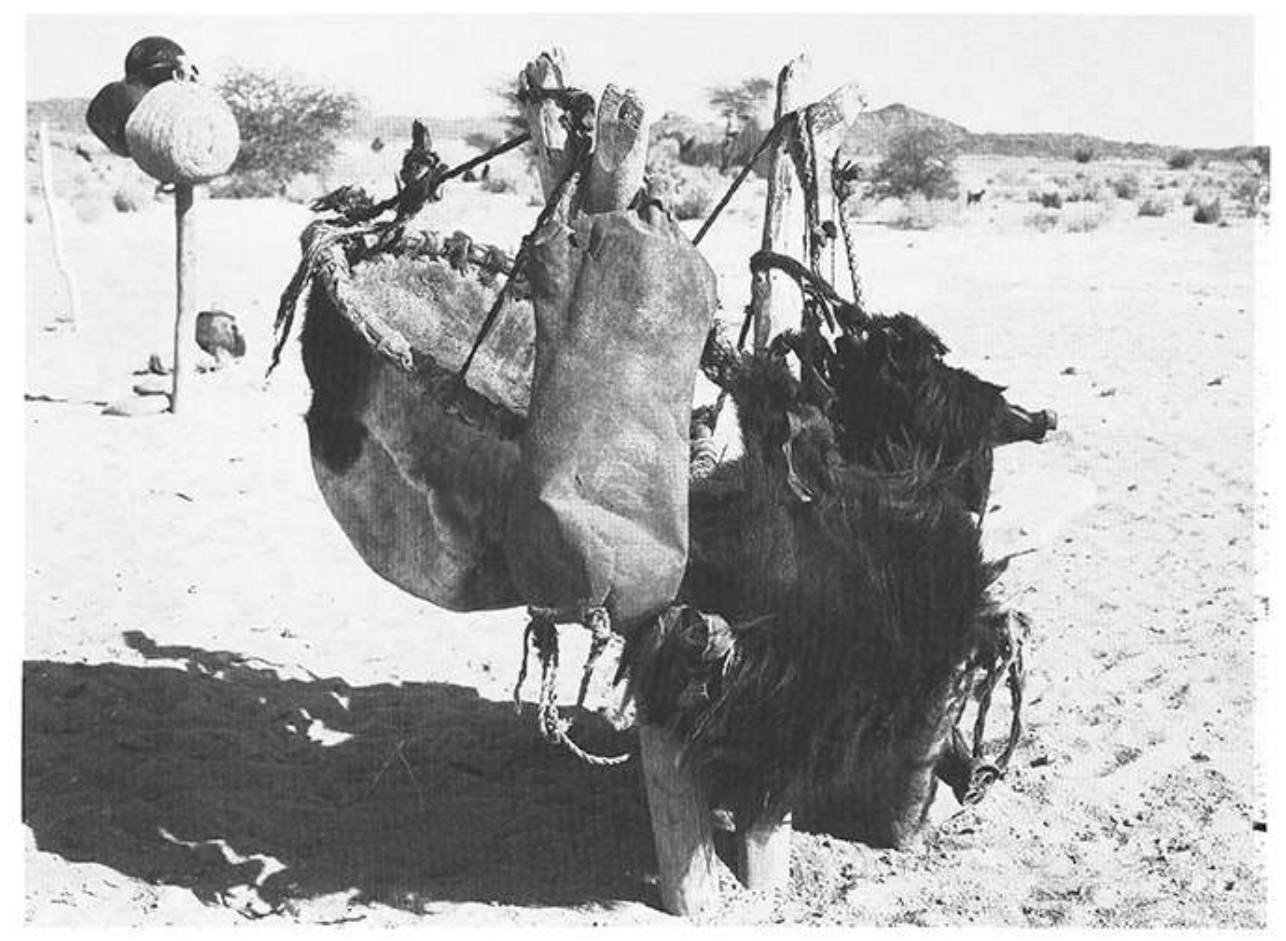

FIg. 1 : OUTRES VIDES et SAC DE PEAU (AHAggAR)

Cliché M. Gast, 1964. 


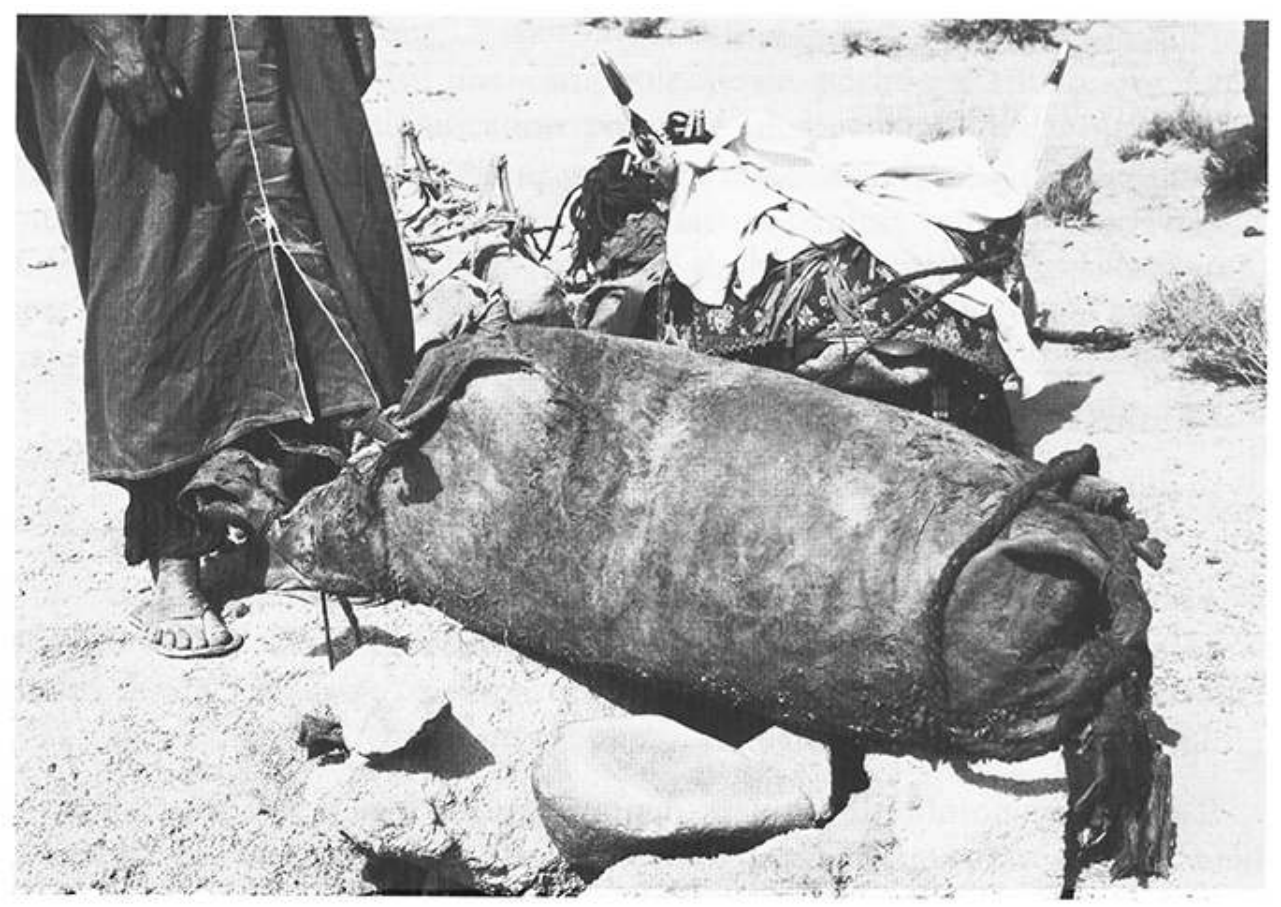

Fig 2 : OUTRE PLEINE de gRAin (IDELÈs, AHAggar)

Cliché M. Gast, 1966.

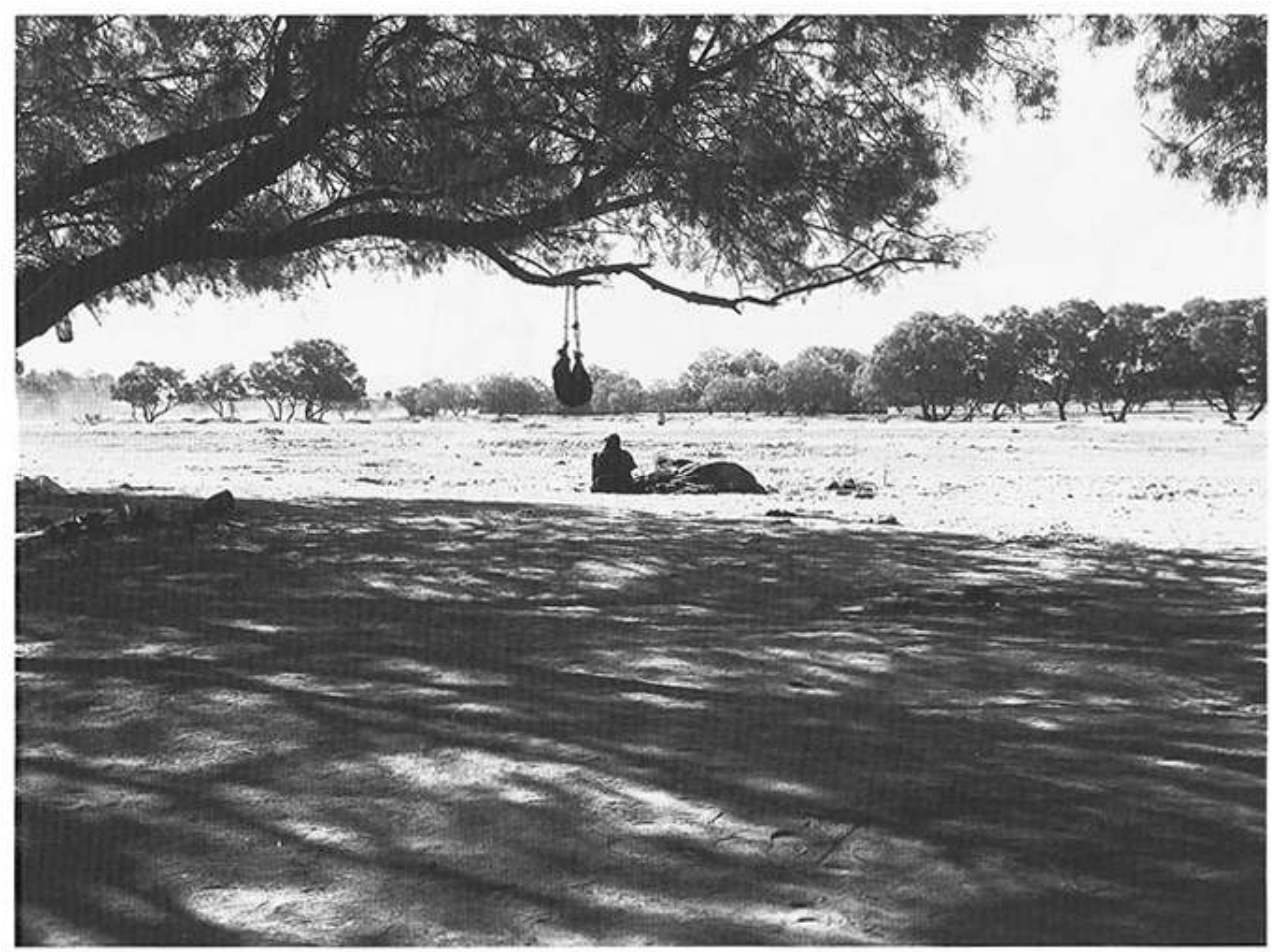

Fig 3 : OUTRE SUSPENDUe À Un ARBRe, OUed tAMANRASSET (NOMAdes du “SOUdAN" CAMPÉs)

Cliché M. Gast, 1961

20 YDD/GDD, «outre (à eau) ». La forme YDD et la signification «outre à eau » sont clairement confirmées par le Zenaga : äydyed (Taine-Cheikh, p. 575).

21 NWR, « outre-baratte à lait » 
YLW / WLK, « outre à provisions » : sans être certain, le lien entre les deux racines peut être envisagé. Une relation de YLW avec la racine YL(W ?), " posséder » (cf. kabyle ayla, "biens, possession », ayl " posséder, avoir droit sur »...) est également possible dans la mesure où taylewt contenait les provisions sèches, principalement les céréales, composante essentielle de la survie alimentaire.

\section{La préparation des peaux et des outres (Kabylie) :}

Témoignage de Lğuhər n Asmər Uhıdduš (grand-mère maternelle de S. Chaker, décédée en 1979 - collecté en 1977 ; transcription et traduction de S.C.).

La description rapportée ici fait référence à des pratiques que l'on peut situer vers 1920, le témoin étant alors âgé d'une dizaine d'années; mais les circonstances déclenchantes de ces souvenirs - le sacrifice d'un bouc pour le creusement des fondations d'une maison - sont bien plus récentes et remontent à la fin des années 1970.

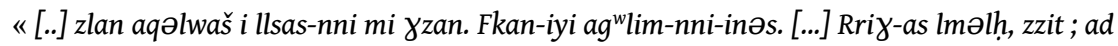
isəw zzit, ad yəqqar akken... rriy-t d tahidurt. [...]

Qqarən-as tagwlla, zzit $d$ wəwrən mi ara yili ur as-tərrid ara lməlh. D tinna umi qqarən tag'lla. Ttarran-tt zik i təyləwt. A s-təkksəḍ tadutț-nni, a t-tərred d tayləwt, ad tərrəd deg-s timzin, irdən...

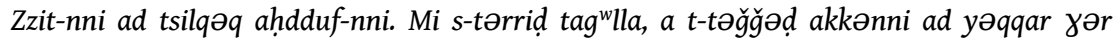
tməss, a t-tšənšwəd ; yəttənšaw imir-n s sshala... udud i s-qqarən. Tayləwt-nni, a tttəgrəḍ di zzit, ad təqqim alamma d tacašurt, a tt-tafə⿳亠丷厂 d tazurant, tag ${ }^{w} l l a-n n i$ tzuzər agw lim-nni. [...]

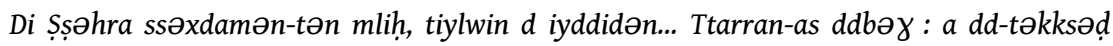
tidəkt, a dd-tnəğrəḍ tasaft, a tən-təddzəḍ akkənni ; ttarran-t $i$ uyddid, ttaran-t $i$ usag ${ }^{w} \partial m$ akkən ur dd-yəggar ara. A tən-tnəydəd, a tən-tərrəd dəgg waman, dya ad rəglən akkw imərğan-nni...

Šfir akka i s-txəddəm yəmma. »

«[...] Ils ont sacrifié un bouc pour les fondations (de la maison) quand ils les ont creusées. Ils m'en ont donné la peau. Je lui ai mis du sel et de l'huile, afin qu'elle boive l'huile et sèche ainsi. J'en ai fait cette peau (pour s'asseoir). [...]

On appelle cela $\operatorname{tag}^{w} l l a(p a ̂ t e 1)$, ce mélange d'huile et de farine quand on n'y met pas de sel. C'est cela que l'on appelle tag ${ }^{w} l l a$ On la mettait autrefois pour (préparer) les outres. On enlève la laine (de la peau) et on en fait une outre dans laquelle on conserve l'orge, le blé...

L'huile assouplit la peau. Quand on lui a appliqué la pâte, on laisse la peau sécher ainsi près $\mathrm{du}$ feu, puis on l'épile; cela s'épile facilement alors... On appelle cela $u d u d^{2}$. Ensuite, on plonge l'outre dans l'huile, on l'y laisse jusqu'à l'Achoura ${ }^{3}$; on retrouve la peau bien épaisse, la pâte ayant épaissi la peau. [...] Au Sahara, on utilise encore beaucoup les outres (à denrées sèches / à eau)...

On leur met un tanin : on prend du lentisque, on taille de l'écorce de chêne et on les pile ensemble; on utilise cette préparation pour les outres (à eau) et pour les amphores (à eau) pour qu'elles ne perdent pas. On met la préparation dans l'eau, alors tous les (petits) trous (de l'outre ou de l'amphore) se bouchent...

Je me souviens que ma mère faisait ainsi. » 


\section{BIBLIOGRAPHIE}

DALLET J.-M., Dictionnaire kabyle-français, Paris, SELAF/Peeters, 1982.

DELHEURE J., Dictionnaire mozabite-français, Paris, SELAF/Peeters, 1984.

DELHEURE J., Dictionnaire ouargli-français, Paris, SELAF/Peeters, 1987.

DESTAING E., Vocabulaire français-berbère (Etude sur la tachelhit du Sous), Paris, Leroux, 1938.

FOUCAULD Ch. de, Dictionnaire touareg-français, 1952.

GAST M., Alimentation des populations de l'Ahaggar. Etude ethnographique, Paris, Arts et Métiers Graphiques, 1968, p. 371 à 388.

HUYGHE G., Dictionnaire français-kabyle, Malines (Belgique), I. \& A. Godenne, 1902-1903.

HUYGHE G., Dictionnaire français-chaouia, Alger, A. Jourdan, 1906.

MERCIER G., Vocabulaire et textes berbères dans le dialecte des Aït Izdeg, Rabat, René Céré, 1937.

NAÏT-ZERRAD K., Dictionnaire des racines berbères (formes attestées), I, II, III, Paris/Louvain Peeters, 1998, 1999, 2002 + base lexicographique.

PRASSE K.-G. et alii, Dictionnaire français-touareg, Museum Tuscululanum Press/University of Copenhague, 2003.

TAINE-CHEIKH C., Dictionnaire zénaga-français, Rüdiger Köppe Verlag (Berber Sudies, 20), Köln, 2008.

\section{NOTES}

1. tag ${ }^{w} l l a$ (var. tagəlla) est un terme pan-berbère dont la signification de base semble être « pâte à pain, pain » (cf. l'expression kabyle tagwlla d lmelh, « partager) le pain et le sel »).

2. udud, désigne précisément cette préparation (pâte à l'huile) dans laquelle a macéré la peau et qui prend rapidement une odeur nauséabonde (cf. Dallet, Dictionnaire kabyle-français, 1982, p. 171). 3. La fête musulmane de l'Achoura* a lieu le $10 \mathrm{du}$ mois de Muharram, soit une quarantaine de jours après l'Aïd el-Kébir, « fête du mouton ", à l'occasion de laquelle on a sacrifié une bête.

\section{INDEX}

Mots-clés : Ethnographie, Ethnolinguistique, Sahara, Touareg 